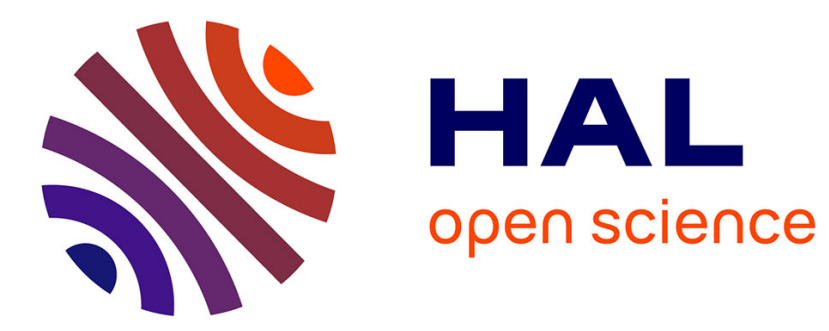

\title{
Theoretical tools for atom-laser-beam propagation
}

Jean-Félix Riou, Yann Le Coq, François Impens, William Guerin, Christian J.

Bordé, Alain Aspect, Philippe Bouyer

\section{To cite this version:}

Jean-Félix Riou, Yann Le Coq, François Impens, William Guerin, Christian J. Bordé, et al.. Theoretical tools for atom-laser-beam propagation. Physical Review A : Atomic, molecular, and optical physics [1990-2015], 2008, 77, pp.033630. 10.1103/PhysRevA.77.033630 . hal-00820911

\section{HAL Id: hal-00820911 \\ https://hal-iogs.archives-ouvertes.fr/hal-00820911}

Submitted on 7 Apr 2016

HAL is a multi-disciplinary open access archive for the deposit and dissemination of scientific research documents, whether they are published or not. The documents may come from teaching and research institutions in France or abroad, or from public or private research centers.
L'archive ouverte pluridisciplinaire HAL, est destinée au dépôt et à la diffusion de documents scientifiques de niveau recherche, publiés ou non, émanant des établissements d'enseignement et de recherche français ou étrangers, des laboratoires publics ou privés. 


\title{
Theoretical tools for atom-laser-beam propagation
}

\author{
J.-F. Riou, ${ }^{1, *}$ Y. Le Coq, ${ }^{2}$ F. Impens, ${ }^{2}$ W. Guerin, ${ }^{1, \dagger}$ C. J. Bordé, ${ }^{2}$ A. Aspect,,${ }^{1}$ and P. Bouyer ${ }^{1}$ \\ ${ }^{1}$ Laboratoire Charles Fabry de l'Institut d'Optique, CNRS et Université Paris Sud 11, Campus Polytechnique, \\ RD 128, 91127 Palaiseau, France \\ ${ }^{2}$ SYRTE, Observatoire de Paris, CNRS, UPMC, 61 avenue de l'Observatoire, 75014 Paris, France
}

(Received 27 December 2007; published 27 March 2008)

\begin{abstract}
We present a theoretical model for the propagation of non-self-interacting atom laser beams. We start from a general propagation integral equation and we use the same approximations as in photon optics to derive tools to calculate the atom-laser-beam propagation. We discuss the approximations that allow one to reduce the general equation whether to a Fresnel-Kirchhoff integral calculated by using the stationary phase method, or to the eikonal. Within the paraxial approximation, we also introduce the $A B C D$ matrices formalism and the beam quality factor. As an example, we apply these tools to analyze the recent experiment by Riou et al. [Phys. Rev.
\end{abstract} Lett. 96, 070404 (2006)].

DOI: 10.1103/PhysRevA.77.033630

PACS number(s): 03.75.Pp, 42.60.Jf, 41.85.Ew

\section{INTRODUCTION}

Matter-wave optics, where a beam of neutral atoms is considered for its wavelike behavior, is a domain of considerable studies, with many applications, ranging from atom lithography to atomic clocks and atom interferometer [1]. The experimental realization of coherent matter wave-socalled atom lasers [2-8] — which followed the observation of Bose-Einstein condensation put a new perspective to the field by providing the atomic analog to photonic laser beams.

Performant theoretical tools for characterizing the propagation properties of matter waves and their manipulation by atom-optics elements are of prime interest for high accuracy applications, as soon as one needs to go beyond the proofof-principle experiment. In the scope of partially coherent atom interferometry, and for relatively simple (i.e., homogenous) external potentials, many theoretical works have been developed [9-12] and applied successfully [13,14]. All these tools essentially address the propagation of an atomic wave packet. For fully coherent atom-laser beams, most theoretical investigations focused on the dynamics of the outcoupling [15-28] and the quantum statistical properties of the output beam [29-36]. Some works specifically addressed the spatial shape of the atom laser beam $[37,38]$, but rely essentially on numerical simulations or neglect the influence of dimensionality and potential inhomogeneity. For realistic experimental conditions, the 3D external potential is inhomogeneous and full numerical simulation becomes particularly cumbersome. One thus needs a simplified analytical theoretical framework to handle the beam propagation.

Following our previous work $[39,40]$, we present here in detail a simple but general framework for the propagation of atom laser beams in inhomogeneous media. We show how several theoretical tools from classical optics can be adapted

\footnotetext{
*Present address: Physics Department of Penn State University, 104 Davey Laboratory, Mailbox 002, University Park, PA 16802. jfriou@ phys.psu.edu

†Present address: Institut Non Linéaire de Nice, 1361 route des Lucioles, 06560 Valbonne, France.
}

for coherent atom optics. We address three major formalisms used in optics: The eikonal approximation, the FresnelKirchhoff integral, and the $A B C D$ matrices formalism in the paraxial approximation.

The first part of the paper gives an overview of these theoretical tools for atom-laser-beam propagation. In the first section, we introduce the integral equation of the propagation and its time-independent version. We present in the second section different ways of dealing with the time-independent propagation of the matter wave. First, the time-independent propagator is computed using the stationary phase approximation. Then, we show that two approximations-the eikonal and the paraxial approximation, which apply in different physical contexts, can provide a more tractable treatment than the general integral equation. In the second part, we show in practice how to use these methods in the experimental case of [39] with a rubidium radiofrequency-coupled atom laser. Some of these methods have recently been used also for a metastable helium atom laser [41] as well as for a Raman-coupled atom laser [42].

\section{ANALYTICAL PROPAGATION METHODS FOR MATTER WAVES}

\section{A. Matter wave weakly outcoupled from a source}

\section{Propagation equation}

We consider a matter wave $\psi_{\ell}(\mathbf{r}, t)$ outcoupled from a source $\psi_{\mathrm{s}}(\mathbf{r}, t)$. We note $V_{i}(\mathbf{r}, t) \quad(i=\{\ell, \mathrm{s}\})$, the external potential in which each of them evolves. We also introduce a coupling term $W_{i j}(\mathbf{r}, t)$ between $\psi_{i}$ and $\psi_{j}$. In the mean-field approximation, such system is described by a set of two coupled Gross-Pitaevskii equations, which reads

$$
i \hbar \partial_{t} \psi_{i}=\left[-\frac{\hbar^{2}}{2 m} \Delta+V_{i}+\sum_{k=\ell, \mathrm{s}} g_{k i}\left|\psi_{k}\right|^{2}\right] \psi_{i}+W_{i j} \psi_{j} .
$$

In this equation, $g_{i k}$ is the mean-field interaction strength between states $i$ and $k$. The solution of such equations is not straightforward, mainly due to the presence of a nonlinear mean-field term. However, in the case of propagation of mat- 
ter waves which are weakly outcoupled from a source, one can greatly simplify the treatment [28]. Indeed, the weakcoupling assumption implies the two following points: The evolution of the source wave function is unaffected by the outcoupler and the extracted matter wave is sufficiently diluted to make self-interactions negligible.

The former differential system can then be rewritten as

$$
\begin{gathered}
i \hbar \partial_{t} \psi_{\mathrm{s}}=\left[-\frac{\hbar^{2}}{2 m} \Delta+V_{\mathrm{s}}+g_{\mathrm{ss}}\left|\psi_{\mathrm{s}}\right|^{2}\right] \psi_{\mathrm{s}}, \\
i \hbar \partial_{t} \psi_{\ell}=\left[-\frac{\hbar^{2}}{2 m} \Delta+V_{\ell}+g_{\mathrm{s} \ell}\left|\psi_{\mathrm{s}}\right|^{2}\right] \psi_{\ell}+W_{\ell \mathrm{s}} \psi_{\mathrm{s}} .
\end{gathered}
$$

The source wave-function $\psi_{\mathrm{s}}(\mathbf{r}, t)$ now obeys a single differential Eq. (2) and can thus be determined independently. The remaining nonlinear term $\left|\psi_{\mathrm{s}}\right|^{2}$ in Eq. (3) acts then as an external potential for the propagation of $\psi_{\ell}$. This last equation is thus a Schrödinger equation describing the evolution of the outcoupled matter wave in the total potential $V(\mathbf{r}, t)$ in the presence of a source term $\rho(\mathbf{r}, t)$,

$$
i \hbar \partial_{t} \psi_{\ell}=H_{\mathbf{r}} \psi_{\ell}+\rho,
$$

where

$$
\begin{gathered}
H_{\mathbf{r}}=-\frac{\hbar^{2}}{2 m} \Delta_{\mathbf{r}}+V, \\
V=V_{\ell}+g_{\mathrm{s} \ell}\left|\psi_{\mathrm{s}}\right|^{2}, \\
\rho=W_{\ell \mathrm{s}} \psi_{\mathrm{s}} .
\end{gathered}
$$

\section{Integral equation}

The evolution between times $t_{0}$ and $t\left(t>t_{0}\right)$ of the solution $\psi_{\ell}$ of Eq. (4) in a given volume $\mathcal{V}$ delimited by a surface $\mathcal{S}$ is expressed by an implicit integral [43]

$$
\begin{aligned}
\psi_{\ell}(\mathbf{r}, t)= & \int_{\mathcal{V}} d \mathbf{r}^{\prime} \mathcal{G}\left(\mathbf{r}, \mathbf{r}^{\prime}, t-t_{0}\right) \psi_{\ell}\left(\mathbf{r}^{\prime}, t_{0}\right) \\
& +\frac{i \hbar}{2 m} \int_{t_{0}}^{t} d t^{\prime} \int_{\mathcal{S}} d \mathbf{S}^{\prime}\left[\mathcal{G}\left(\mathbf{r}, \mathbf{r}^{\prime}, t-t^{\prime}\right) \nabla_{\mathbf{r}^{\prime}} \psi_{\ell}\left(\mathbf{r}^{\prime}, t^{\prime}\right)\right. \\
& \left.-\psi_{\ell}\left(\mathbf{r}^{\prime}, t^{\prime}\right) \nabla_{\mathbf{r}^{\prime}} \mathcal{G}\left(\mathbf{r}, \mathbf{r}^{\prime}, t-t^{\prime}\right)\right] \\
& +\frac{1}{i \hbar} \int_{t_{0}}^{t} d t^{\prime} \int_{\mathcal{V}} d \mathbf{r}^{\prime} \mathcal{G}\left(\mathbf{r}, \mathbf{r}^{\prime}, t-t^{\prime}\right) \rho\left(\mathbf{r}^{\prime}, t^{\prime}\right),
\end{aligned}
$$

where $d \mathbf{S}^{\prime}$ is the outward-oriented elementary normal vector to the surface $\mathcal{S}$. We have introduced the time-dependent Green function $\mathcal{G}\left(\mathbf{r}, \mathbf{r}^{\prime}, \tau\right)$ which verifies

$$
\left[i \hbar \partial_{\tau}-H_{\mathbf{r}}\right] \mathcal{G}=i \hbar \delta(\tau) \delta\left(\mathbf{r}-\mathbf{r}^{\prime}\right)
$$

and is related to the propagator $\mathcal{K}$ of the Schrödinger equation via a Heaviside function $\Theta$ ensuring causality,

$$
\mathcal{G}\left(\mathbf{r}, \mathbf{r}^{\prime}, \tau\right)=\mathcal{K}\left(\mathbf{r}, \mathbf{r}^{\prime}, \tau\right) \Theta(\tau) .
$$

Equation (6) states that, after the evolution time $t-t_{0}$, the value of the wave function is the sum of three terms, the physical interpretation of which is straightforward. The first one corresponds to the propagation of the initial condition $\psi_{\ell}\left(\mathbf{r}^{\prime}, t_{0}\right)$ given at any position in the volume $\mathcal{V}$. The second one takes into account the propagation of the wave function taken at the surrounding surface $\mathcal{S}$ and is nonzero only if $\mathcal{V}$ is finite. This term takes into account any field which enters or leaks out of $\mathcal{V}$. Finally, the last term expresses the contribution from the source.

Equation (6) can be successfully applied to describe the propagation of wave packets in an atom interferometer as described in [44]. Nevertheless, the propagation of a continuous atom laser, the energy of which is well defined, can be described with a time-independent version of Eq. (6), that we derive below.

\section{Time-independent case}

We consider a time-independent Hamiltonian $H_{\mathbf{r}}$ and a stationary source

$$
\rho(\mathbf{r}, t)=\rho(\mathbf{r}) \exp (-i E t / \hbar) .
$$

We thus look for stationary solutions of Eq. (4) with a given energy $E$,

$$
\psi_{\ell}(\mathbf{r}, t)=\psi_{\ell}(\mathbf{r}) \exp (-i E t / \hbar) .
$$

When $t_{0} \rightarrow-\infty$, Eq. (6) then becomes time independent:

$$
\begin{aligned}
\psi_{\ell}(\mathbf{r})= & \frac{1}{i \hbar} \int_{\mathcal{V}} d \mathbf{r}^{\prime} \mathcal{G}_{E}\left(\mathbf{r}, \mathbf{r}^{\prime}\right) \rho\left(\mathbf{r}^{\prime}\right) \\
& +\frac{i \hbar}{2 m} \int_{\mathcal{S}} d \mathbf{S}^{\prime}\left[\mathcal{G}_{E}\left(\mathbf{r}, \mathbf{r}^{\prime}\right) \nabla_{\mathbf{r}^{\prime}} \psi_{\ell}\left(\mathbf{r}^{\prime}\right)\right. \\
& \left.-\psi_{\ell}\left(\mathbf{r}^{\prime}\right) \nabla_{\mathbf{r}^{\prime}} \mathcal{G}_{E}\left(\mathbf{r}, \mathbf{r}^{\prime}\right)\right]
\end{aligned}
$$

where $\mathcal{G}_{E}$ is the time-independent propagator related to $\mathcal{K}$ via

$$
\mathcal{G}_{E}\left(\mathbf{r}, \mathbf{r}^{\prime}\right)=\int_{0}^{+\infty} d \tau \mathcal{K}\left(\mathbf{r}, \mathbf{r}^{\prime}, \tau\right) e^{i E \tau / \hbar}
$$

Note that the first term of Eq. (6) vanishes in the timeindependent version of the propagation integral equation as $\mathcal{K}\left(\mathbf{r}, \mathbf{r}^{\prime}, \tau\right) \rightarrow 0$ when $\tau \rightarrow \infty$. The second term of Eq. (11) is the equivalent for matter waves of what is known in optics as the Fresnel-Kirchhoff integral [45].

\section{B. Major approximations for atom-laser-beam propagation}

\section{Independent treatment of a succession of potentials}

As an optical wave can enter different media (free space, lenses, etc.) separated by surfaces, matter waves can propagate in different parts of space, where they experience potentials of different nature. For instance, when one considers an atom laser outcoupled from a condensate as in the example of Sec. III, the beam initially interacts with the Bosecondensed atoms and abruptly propagates in free space outside of the condensate. The expression of the propagator in whole space would then be needed to use the Eq. (11). Most generally, such calculation requires to apply the Feynmann's path integral method, either numerically or analytically [46]. 
For example, the time-dependent propagator $\mathcal{K}$ can be analytically expressed in the case of a continuous potential which is at most quadratic, by using Van Vleck's formula [47] or the $A B C D$ formalism [44]. However, such expressions fail to give the global propagator value for a piecewisedefined quadratic potential.

As in classical optics, we can separate the total evolution of a monochromatic wave in steps, each one corresponding to one homogeneous potential. This step-by-step approach stays valid as long as one can neglect any reflection on the interface between these regions as well as feedback from one region to a previous one. In this approach, each interface is considered as a surface source term for the propagation in the following media. It allows us to calculate $\mathcal{K}$ explicitly in every part of space as long as the potential in each region remains at most quadratic, which we will assume throughout this paper.

\section{Time-independent propagator in the stationary phase approximation}

Whereas the expression of $\mathcal{G}_{E}$ is well known for free space and linear potentials [9,38], to our knowledge, there is no analytical expression for the inverted harmonic potential, which plays a predominant role in an atom laser interacting with its source condensate. We thus give in the following a method to calculate the time-independent propagator $\mathcal{G}_{E}$ in any up to quadratic potential.

Since $\mathcal{K}$ is analytically known in such potentials, we use the definition of $\mathcal{G}_{E}$ as its Fourier transform [Eq. (12)]. The remaining integral over time $\tau$ is calculated via a stationary phase method [45], taking advantage that $\mathcal{K}$ is a rapidly oscillating function. We write the time-dependent propagator as

$$
\mathcal{K}\left(\mathbf{r}, \mathbf{r}^{\prime}, \tau\right)=\mathcal{A}(\tau) \exp \left[i \phi\left(\mathbf{r}, \mathbf{r}^{\prime}, \tau\right)\right] .
$$

We introduce $\tau_{n}$ as the positive real solution(s) of

$$
\partial_{\tau} \phi\left(\mathbf{r}, \mathbf{r}^{\prime}, \tau_{n}\right)=-E / \hbar,
$$

which correspond(s) to the time(s) spent on classical path(s) of energy $E$ connecting $\mathbf{r}^{\prime}$ to $\mathbf{r}$. We develop $\phi$ to the second order around $\tau_{n}$,

$$
\phi(\tau) \simeq \phi\left(\tau_{n}\right)+\left.\frac{\partial \phi}{\partial \tau}\right|_{\tau_{n}}\left(\tau-\tau_{n}\right)+\left.\frac{\partial^{2} \phi}{\partial \tau^{2}}\right|_{\tau_{n}} \frac{\left(\tau-\tau_{n}\right)^{2}}{2} .
$$

Using the last development in the integral (12), and assuming that the envelope $\mathcal{A}(\tau)$ varies smoothly around $\tau_{n}$, we can express $\mathcal{G}_{E}$ as

$$
\mathcal{G}_{E}^{(1)} \simeq \sum_{n} \sqrt{\frac{2 i \pi}{\phi^{\prime \prime}\left(\tau_{n}\right)}} \mathcal{K}\left(\tau_{n}\right) \exp \left(i \frac{E \tau_{n}}{\hbar}\right) .
$$

Such an approach is valid as long as stationary points $\tau_{n}$ exist and their contribution can be considered independently: Eq. (16) fails if the stationary points are too close to each other. We can estimate the validity of our approach by defining an interval $\mathcal{I}_{n}=\left[\tau_{n}-\theta_{n} ; \tau_{n}+\theta_{n}\right]$ in which the development around $\tau_{n}$ contributes to more than $\beta=90 \%$ to the restricted integral. For $\theta$ large enough, we can use [48]

$$
\left|\int_{-\theta}^{\theta} d x \exp \left[i z \frac{x^{2}}{2}\right]-\sqrt{\frac{2 i \pi}{z}}\right| \sim \frac{2}{|z \theta|},
$$

and obtain $\theta_{n}$

$$
\theta_{n}=\frac{1}{1-\beta} \sqrt{\frac{2}{\pi \phi^{\prime \prime}\left(\tau_{n}\right)}} .
$$

The validity condition is thus $\left|\tau_{n}-\tau_{n+1}\right| \geq \theta_{n}+\theta_{n+1}$.

If Eq. (16) is not valid, a better approximation consists then in developing $\phi$ to higher order around a point which is in between successive $\tau_{n}$. The simplest choice is to take the one which cancels $\phi^{\prime \prime}$ and to choose stationary points $\tau_{k}$ which verify

$$
\partial_{\tau}^{2} \phi\left(\mathbf{r}, \mathbf{r}^{\prime}, \tau_{k}\right)=0 .
$$

We thus develop $\phi$ to the third order around $\tau_{k}$, which leads to the following expression of $\mathcal{G}_{E}$,

$$
\mathcal{G}_{E}^{(2)} \simeq \frac{2 \pi \mathcal{K}\left(\tau_{k}\right) \exp \left(i \frac{E \tau_{k}}{\hbar}\right)}{\sqrt[3]{-\phi^{(3)}\left(\tau_{k}\right) / 2}} \operatorname{Ai}\left(\frac{\phi^{\prime}\left(\tau_{k}\right)+E / \hbar}{\sqrt[3]{\phi^{(3)}\left(\tau_{k}\right) / 2}}\right),
$$

where $\mathrm{Ai}$ is the Airy function of the first kind [49].

In practice, combining the use of $\mathcal{G}_{E}^{(2)}$ and $\mathcal{G}_{E}^{(1)}$ depending on the values of $\mathbf{r}^{\prime}$ and $\mathbf{r}$ gives a good estimate of the timeindependent propagator, as we will see in Sec. III.

Although the above approach is quite general, further approximations can be made. In the region where diffraction can be neglected, one can describe the propagation with the eikonal approximation. When the propagation is in the paraxial regime, it is more appropriate to describe it with the paraxial $A B C D$ matrices, instead of using the general Kirchhoff integral.

\section{Eikonal propagation}

The purpose of this method, equivalent to the WKB approximation, is to give a semiclassical description of the propagation from a matter wave, given its value on a surface. Let us consider that we know the value of the wave function of energy $E$ on the surface $\mathcal{S}^{\prime}$. To calculate its value on any other surface $\mathcal{S}$, the eikonal considers classical paths connecting $\mathcal{S}$ and $\mathcal{S}^{\prime}$. Let us write the wave function as

$$
\psi_{\ell}(\mathbf{r})=A(\mathbf{r}) \exp [i S(\mathbf{r}) / \hbar] .
$$

The Schrödinger equation on $\psi_{\ell}$ reduces to [50]

$$
\left|\nabla_{\mathbf{r}} S\right|=\frac{\hbar}{\chi}, \quad \nabla_{\mathbf{r}}\left(A^{2} \nabla S\right)=0,
$$

where we have introduced the de Broglie wavelength

$$
\chi(\mathbf{r})=\frac{\hbar}{\sqrt{2 m[E-V(\mathbf{r})]}} .
$$

The first equation is known in geometric optics as the eikonal equation $[45,51]$. The calculation consists in integrating the phase along the classical ray of energy $E$ connecting $\mathbf{r}^{\prime}$ to $\mathbf{r}$, to obtain the phase on $\mathbf{r}$, 


$$
S(\mathbf{r})=\int_{\mathbf{r}^{\prime}}^{\mathbf{r}} d \mathbf{u} \frac{\hbar}{\chi(\mathbf{u})}+S\left(\mathbf{r}^{\prime}\right)
$$

The second equation of system (22) corresponds to the conservation of probability density flux and is equivalent to the Poynting's law in optics. Again, after integration along the classical path connecting $\mathbf{r}^{\prime}$ to $\mathbf{r}$, one obtains the amplitude on $\mathcal{S}$

$$
A(\mathbf{r})=A\left(\mathbf{r}^{\prime}\right) \exp \left(-\int_{\mathbf{r}^{\prime}}^{\mathbf{r}} d \mathbf{u} \frac{\Delta S(\mathbf{u}) \chi(\mathbf{u})}{2 \hbar}\right)
$$

Note that interference effects are included in this formalism: If several classical paths connect $\mathbf{r}^{\prime}$ to $\mathbf{r}$, their respective contributions add coherently to each other. Also, if some focusing points exist, dephasings equivalent to the Gouy phase in optics appear and can be calculated following [45].

Such semiclassical treatment is valid as long as one does not look for the wave function value close to classical turning points, and as long as transverse diffraction is negligible: transverse structures of size $\Delta x$ must be large enough not to diffract significantly, i.e., $\Delta x^{4} \gg(\hbar t / 2 m)^{2}$. This condition restricts the use of the eikonal to specific regions of space where the matter wave does not spend a too long time $t$. For instance, this is the case for the propagation in the small region of overlap with the BEC.

The eikonal can thus be used to deal with the first term of Eq. (11) and is equivalent to the development of this integral around classical trajectories [52].

\section{Paraxial propagation}

The paraxial regime applies as soon as the transverse wave vector becomes negligible compared to the axial one. It is for instance the case after some propagation for gravityaccelerated atom-laser beams. We can then take advantage of methods developed in optics and use the paraxial atomoptical $A B C D$ matrices formalism [40,44], instead of the general Kirchhoff integral, and characterize globally the beam with the quality factor $M^{2}[53,54]$.

(a) The paraxial equation. We look for paraxial solutions to the time-independent Schrödinger equation,

$$
H_{\mathbf{r}} \psi_{\ell}(\mathbf{r})=E \psi_{\ell}(\mathbf{r})
$$

We decompose the wave function and the potential in a transverse (" $\perp$ ") and parallel ("I") component, taking $z$ as the propagation axis,

$$
\psi_{\ell}(x, y, z)=\psi_{\perp}(x, y, z) \psi_{\|}(z), \quad V(x, y, z)=V_{\perp}(x, y, z)+V_{\|}(z),
$$

where $V_{\|}(z)=V(0,0, z)$. We express the solution $\psi_{\|}$to the one-dimensional equation

$$
-\frac{\hbar^{2}}{2 m} \frac{\partial^{2} \psi_{\|}}{\partial z^{2}}+V_{\|} \psi_{\|}=E \psi_{\|}
$$

by using the WKB approximation

$$
\psi_{\|}(z)=\sqrt{\frac{m \mathcal{F}}{p(z)}} \exp \left[\frac{i}{\hbar} \int_{z_{0}}^{z} d u p(u)\right]
$$

In this expression, $\mathcal{F}$ is the atomic flux through any transverse plane, $p(z)=\sqrt{2 m\left[E-V_{\|}(z)\right]}$ is the classical momentum along $z$ and $z_{0}$ is the associated classical turning point verifying $p\left(z_{0}\right)=0$. Using these expressions, and assuming an envelope $\psi_{\perp}$ slowly varying along $z$, we obtain the paraxial equation of propagation for the transverse profile,

$$
\left[i \hbar \partial_{\zeta}+\frac{\hbar^{2}}{2 m}\left(\partial_{x}^{2}+\partial_{y}^{2}\right)-V_{\perp}(x, y, \zeta)\right] \psi_{\perp}(x, y, \zeta)=0,
$$

where $\zeta(z)=\int_{z_{0}}^{z} d z \mathrm{~m} / p(z)$ is a parameter corresponding to the time which would be needed classically to propagate on axis from the turning point $z_{0}$. Equation (30) can thus be solved as a time-dependent Schrödinger equation,

$$
\psi_{\perp}(x, y, \zeta)=\int_{\mathcal{S}^{\prime}} d x^{\prime} d y^{\prime} \mathcal{K}\left(x, y ; x^{\prime}, y^{\prime} ; \zeta-\zeta^{\prime}\right) \psi_{\perp}\left(x^{\prime}, y^{\prime}, \zeta^{\prime}\right) \text {. }
$$

The use of the paraxial approximation allows us to focus only on the evolution of the transverse wave function, reducing the dimensionality of the system from $3 \mathrm{D}$ to $2 \mathrm{D}$, as the third dimension along the propagation axis $z$ is treated via a semiclassical approximation [Eq. (29)].

(b) $A B C D$ matrices. In the case of a separable transverse potential independent of $z$, the paraxial approximation restricts to two independent one-dimensional equations. Let us consider a potential $V_{x}$ at most quadratic in $x$. One can then write the propagator $\mathcal{K}_{x}$ by using the Van Vleck formula, or equivalently the general $A B C D$ matrix formalism [9],

$$
\mathcal{K}_{x}=\sqrt{\frac{\alpha}{2 \pi i B}} \exp \left[\frac{i \alpha}{2 B}\left(A x^{\prime 2}+D x^{2}-2 x x^{\prime}\right)\right] .
$$

The coefficients $A, B, C, D$ verifying $A D-B C=1$ are functions of $\zeta-\zeta^{\prime}$ and $\alpha$ is an arbitrary factor depending on the definition of the $A B C D$ coefficients. These ones are involved in the matrix describing the classical dynamics of a virtual particle of coordinate $X$ and speed $V$ in the potential $V_{x}(X)$

$$
\left(\begin{array}{c}
X(\zeta) \\
\alpha V(\zeta)
\end{array}\right)=\left(\begin{array}{cc}
A\left(\zeta-\zeta^{\prime}\right) & B\left(\zeta-\zeta^{\prime}\right) / \alpha \\
\alpha C\left(\zeta-\zeta^{\prime}\right) & D\left(\zeta-\zeta^{\prime}\right)
\end{array}\right)\left(\begin{array}{c}
X\left(\zeta^{\prime}\right) \\
\alpha V\left(\zeta^{\prime}\right)
\end{array}\right)
$$

Different choices of $\alpha$ can be made and popular values in the atomoptic literature are $\alpha=1$ [9] or $\alpha=m / \hbar[39,40]$. We take the last convention and, by introducing the wave vector $K$ $=m V / \hbar$, use throughout this paper the following definition for the $A B C D$ coefficients in which is included the value of $\alpha$,

$$
\left(\begin{array}{l}
X(\zeta) \\
K(\zeta)
\end{array}\right)=\left(\begin{array}{ll}
A\left(\zeta-\zeta^{\prime}\right) & B\left(\zeta-\zeta^{\prime}\right) \\
C\left(\zeta-\zeta^{\prime}\right) & D\left(\zeta-\zeta^{\prime}\right)
\end{array}\right)\left(\begin{array}{l}
X\left(\zeta^{\prime}\right) \\
K\left(\zeta^{\prime}\right)
\end{array}\right) .
$$

(c) Propagation using the Hermite-Gauss basis. To calculate the propagation along the $x$ axis 


$$
\psi_{x}(x, \zeta) \int d x^{\prime} \mathcal{K}_{x}\left(x ; x^{\prime} ; \zeta-\zeta^{\prime}\right) \psi_{x}\left(x^{\prime}, \zeta^{\prime}\right)
$$

it is useful to use the Hermite-Gauss basis of functions $\left(\Phi_{n}\right)_{n \in \mathbb{N}}$

$$
\begin{gathered}
\Phi_{0}(x,\{X, K\})=\frac{(2 \pi)^{-1 / 4}}{\sqrt{X}} \exp \left(i \frac{K}{X} \frac{x^{2}}{2}\right), \\
\Phi_{n}(x,\{X, K\})=\Phi_{0}(x) \frac{1}{\sqrt{2^{n} n !}} \frac{|X|^{n}}{X^{n}} H_{n}\left[\frac{x}{\sqrt{2}|X|}\right] .
\end{gathered}
$$

$H_{n}$ is the $n$th order Hermite polynomial and the two parameters $(X, K) \in \mathrm{C}$, which define univocally the basis set, must verify the normalization condition

$$
K X^{*}-K^{*} X=i
$$

so that this basis is orthonormalized.

These functions propagate easily via $\mathcal{K}_{x}$, as

$$
\Phi_{n}[x,\{X, K\}(\zeta)]=\int d x^{\prime} \mathcal{K}_{x}\left(x ; x^{\prime} ; \zeta-\zeta^{\prime}\right) \Phi_{n}\left[x^{\prime},\{X, K\}\left(\zeta^{\prime}\right)\right]
$$

i.e., the integral is calculated by replacing $X\left(\zeta^{\prime}\right)$ and $K\left(\zeta^{\prime}\right)$ by their value at $\zeta$ through the algebraic relation (34).

Thus the propagation of the function $\psi_{x}$ between two positions $z\left(\zeta^{\prime}\right)$ and $z(\zeta)$ is obtained by first decomposing the initial profile on the Hermite-Gauss basis

$$
\psi_{x}\left(x, \zeta^{\prime}\right)=\sum_{n} c_{n} \Phi_{n}\left[x,\{X, K\}\left(\zeta^{\prime}\right)\right]
$$

where

$$
c_{n}=\int d x \Phi_{n}^{*}\left[x,\{X, K\}\left(\zeta^{\prime}\right)\right] \psi_{x}\left(x, \zeta^{\prime}\right)
$$

The profile after propagation until $z(\zeta)$ is then

$$
\psi_{x}(x, \zeta)=\sum_{n} c_{n} \Phi_{n}[x,\{X, K\}(\zeta)]
$$

The high efficiency of this method comes from the fact that, once the decomposition (40) is made, the profile at any position $z(\zeta)$ is obtained by calculating an algebraic evolution equation: the $A B C D$ law [Eq. (34)]. Such computational method is then much faster than the use of the Kirchhoff integral, which would need to calculate an integral for each considered position.

Note that the initial choice of $\{X, K\}\left(\zeta^{\prime}\right)$ is a priori arbitrary as soon as it verifies the normalization condition (38). However, one can minimize the number of functions $\Phi_{n}$ needed for the decomposition if one chooses $\{X, K\}\left(\zeta^{\prime}\right)$ as a function of the second-order moments of the profile.

(d) Moments and quality factor. Let us define the secondorder moments of $\psi_{x}$,

$$
\left\langle x x^{*}\right\rangle=\int d x x^{2} \psi_{x} \psi_{x}^{*}
$$

$$
\left\langle k k^{*}\right\rangle=\int d x \partial_{x} \psi_{x} \partial_{x} \psi_{x}^{*}
$$

$$
\left\langle x k^{*}+x^{*} k\right\rangle=i \int d x \quad x\left[\psi_{x} \partial_{x} \psi_{x}^{*}-\psi_{x}^{*} \partial_{x} \psi_{x}\right]
$$

where we have used that $\psi_{x}$ is normalized $\left(\int d x\left|\psi_{x}\right|^{2}=1\right)$. We also define the wavefront curvature $\mathcal{C}[55]$ as

$$
\mathcal{C}=\frac{\left\langle x k^{*}+x^{*} k\right\rangle}{2\left\langle x x^{*}\right\rangle}
$$

The three moments follow also an $A B C D$ law during propagation. By introducing the matrix

$$
\mathcal{M}(\zeta)=\left(\begin{array}{cc}
\left\langle x x^{*}\right\rangle & \left\langle x k^{*}+x^{*} k\right\rangle / 2 \\
\left\langle x k^{*}+x^{*} k\right\rangle / 2 & \left\langle k k^{*}\right\rangle
\end{array}\right),
$$

this law is expressed as

$$
\mathcal{M}(\zeta)=\left(\begin{array}{ll}
A & B \\
C & D
\end{array}\right) \mathcal{M}\left(\zeta^{\prime}\right)\left(\begin{array}{ll}
A & B \\
C & D
\end{array}\right)^{t} .
$$

This relation allows us to derive propagation laws on the wavefront second-order moments, such as the rms transverse size (Rayleigh law). As $\operatorname{det}(\mathcal{M})$ is constant, this law also exhibits an invariant of propagation, the beam quality factor $M^{2}$, related to the moments and curvature by

$$
\left\langle x x^{*}\right\rangle\left(\left\langle k k^{*}\right\rangle-\mathcal{C}^{2}\left\langle x x^{*}\right\rangle\right)=\left(\frac{M^{2}}{2}\right)^{2} .
$$

The physical meaning of the $M^{2}$ factor becomes clear by taking the last equation at the waist, i.e., where the curvature $\mathcal{C}$ is zero:

$$
\sqrt{\left\langle x x^{*}\right\rangle_{0}\left\langle k k^{*}\right\rangle_{0}}=\frac{M^{2}}{2}
$$

The $M^{2}$ factor is given by the product of the spatial and momentum widths at the beam waist and indicates how far the beam is from the diffraction limit. Because of the Heisenberg uncertainty relation, the $M^{2}$ factor is always larger than one and equals unity only for a perfect Gaussian wavefront.

Finally, the determination of the second order moments and the $M^{2}$ factor from an initial profile allows us to choose the more appropriate values of $\left\{X\left(\zeta^{\prime}\right), K\left(\zeta^{\prime}\right)\right\}$ to parametrize the Hermite-Gauss basis used for the decomposition at $z\left(\zeta^{\prime}\right)$ [Eq. (40)]. Indeed, these parameters are closely related to the second order moments of the Hermite-Gauss functions $\Phi_{n}(x,\{X, K\})$ by

$$
\begin{gathered}
\left\langle x x^{*}\right\rangle_{\Phi_{n}}=(2 n+1)|X|^{2}, \\
\left\langle k k^{*}\right\rangle_{\Phi_{n}}=(2 n+1)|K|^{2}, \\
\left\langle x k^{*}+x^{*} k\right\rangle_{\Phi_{n}}=(2 n+1)\left(X K^{*}+X^{*} K\right) .
\end{gathered}
$$

From this we obtain that the $M^{2}$ factor of the mode $\Phi_{n}$ is $M_{\Phi_{n}}^{2}=(2 n+1)$ and that all the modes have the same curvature 


$$
\mathcal{C}_{\Phi_{n}}=\mathcal{C}=\left(X K^{*}+X^{*} K\right) / 2\left|X^{2}\right| .
$$

It is thus natural to choose the parameters $\{X, K\}$, so that the curvature of the profile [Eq. (46)] equals $\mathcal{C}$. This last condition, together with the choice $|X|^{2}=\left\langle x x^{*}\right\rangle / M^{2}$, the normalization condition (38) and the choice of $X$ real (the phase of $X$ is a global phase over the wavefront), lead to the univocal determination of the parameters $\left\{X\left(\zeta^{\prime}\right), K\left(\zeta^{\prime}\right)\right\}$ associated with the Hermite-Gauss basis, so that the decomposition of the initial profile $\psi_{x}\left(x, \zeta^{\prime}\right)$ needs a number of terms of the order of $M^{2}$.

\section{APPLICATION TO A RADIOFREQUENCY- OUTCOUPLED ATOM LASER}

We apply the previous framework to the radiofrequency (rf) outcoupled atom laser described in [39] where a BoseEinstein condensate (BEC) of rubidium 87 (mass $m$ ) is magnetically harmonically trapped (frequencies $\omega_{x}=\omega_{z}=\omega_{\perp}$ and $\left.\omega_{y}\right)$ in the ground state $\left|F=1, m_{F}=-1\right\rangle$, and is weakly outcoupled to the untrapped state $\left|F=1, m_{F}=0\right\rangle$. The BEC is considered in the Thomas-Fermi (TF) regime described by the time-independent wave function $\phi_{\mathrm{s}}(r)$, with a chemical potential $\mu$ and TF radii $R_{\perp, y}=\sqrt{2 \mu / m \omega_{\perp, y}^{2}}$ [56]. The external potential experienced by the beam is written

$$
V_{\mathrm{i}}(r)=\mu-\frac{1}{2} m \omega_{\perp}^{2} \sigma^{2}-\frac{1}{2} m\left[\omega_{\perp}^{2}\left(x^{2}+z^{2}\right)+\omega_{y}^{2} y^{2}\right]
$$

inside the $\mathrm{BEC}$ region and

$$
V_{\mathrm{o}}(r)=\frac{1}{2} m \omega^{2} \sigma_{q}^{2}-\frac{1}{2} m \omega^{2}\left[x^{2}+\left(z+\sigma_{q}\right)^{2}\right]
$$

outside. The expulsive quadratic potential of $V_{\mathrm{i}}$ originates from the mean-field interaction (independent of the Zeeman substates for ${ }^{87} \mathrm{Rb}$ ) between the laser and the condensate, whereas that of $V_{\mathrm{o}}$ (frequency $\omega$ ) is due to the second order Zeeman effect. We have noted $\sigma=g / \omega_{\perp}^{2}$ and $\sigma_{q}=g / \omega^{2}$, the vertical sags due to gravity $-m g z$ for $m_{F}=-1$ and $m_{F}=0$ states, respectively. The rf coupling (of Rabi frequency $\Omega_{R}$ ) between the condensate and the beam is considered to have a negligible momentum transfer and provides the atom-laser wave function with a source term $\rho=\hbar \Omega_{R} / 2 \phi_{\mathrm{s}}(r)$.

In the following, we consider a condensate elongated along the $y$ axis $\left(\omega_{\perp} \gg \omega_{y}\right)$, so that the laser dynamics is negligible along this direction $[57,58]$. We thus study independently the evolution in each vertical $(x, z)$ plane at position $y_{0}$. We calculate the beam wave function in two steps corresponding to a propagation in each region defined by $V_{\mathrm{i}}$ and $V_{\mathrm{o}}$ (see Fig. 1). The wave function at the BEC frontier is calculated in Sec. III A using the eikonal approximation. Then, in Sec. III B, we calculate the wave function at any position outside the BEC, with the help of the FresnelKirchhoff formalism and the paraxial $A B C D$ matrices.

\section{A. Propagation in the condensate zone}

In this section, we determine the beam wave function $\psi_{\ell}(\mathbf{r})$ in the condensate zone by using the eikonal formalism described in Sec. II B 3. This formalism is appropriate in this

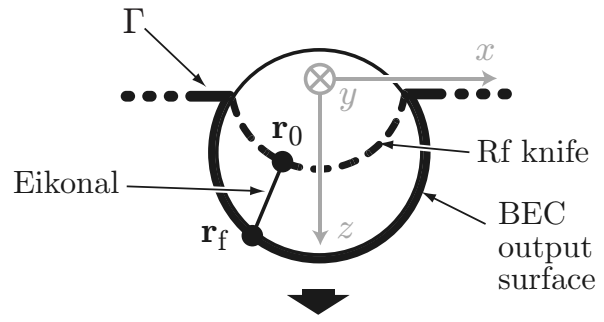

Kirchhoff Int.

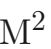

$\mathrm{ABCD}$ matrix

FIG. 1. Principle of the calculation: The wave function is calculated from the rf knife (a circle of radius $r_{0}$ centered at the frame origin) using the eikonal. A general radial atomic trajectory starting at zero speed from $\mathbf{r}_{0}$ crosses the BEC border at $\mathbf{r}_{\mathbf{f}}$. Once the matter wave has exited the condensate region, the wave function is given by the Fresnel-Kirchhoff integral, allowing one to compute the wave function at any point from the BEC output surface. In the paraxial approximation, we calculate the propagation using $A B C D$ matrices.

case as the time necessary for the laser to exit the BEC region $(\approx 1 \mathrm{~ms})$ is small enough so that the transverse diffraction is negligible (transverse size $\approx R_{\perp}$ ).

\section{Atomic rays inside the $B E C$}

One first needs to calculate the atomic paths followed by the atom laser rays from the outcoupling surface (the $\mathrm{rf}$ knife) to the border of the BEC. The rf knife is an ellipsoïd centered at the magnetic field minimum (chosen in the following as the frame origin; see Fig. 1). Its intersection with the $(x, z)$ plane at position $y_{0}$ is a circle centered at the frame origin. Its radius $r_{0}$ depends on the $\mathrm{rf}$ detuning $\delta \nu$ $=(m / 2 h)\left[\omega_{\perp}^{2}\left(r_{0}^{2}-\sigma^{2}\right)+\omega_{y}^{2} y_{0}^{2}\right]$. As we neglect axial dynamics and consider zero initial momentum, the classical equations of motion give for the radial coordinate $r=\sqrt{x^{2}+z^{2}}$, $r(t)=r_{0} \cosh \omega_{\perp} t$ allowing one to find a starting point $\mathbf{r}_{0}$ on the rf knife for each point $\mathbf{r}_{\mathrm{f}}$ on the BEC output surface, i.e., the BEC border below the rf knife [59].

\section{Eikonal expression of the wave function}

We now introduce

$$
a_{\perp}=\sqrt{\frac{\hbar}{m \omega_{\perp}}}, \quad R=\frac{r}{a_{\perp}}, \quad \epsilon=-\left(\frac{r_{0}}{a_{\perp}}\right)^{2},
$$

which are respectively the size of the harmonic potential, the dimensionless coordinate, and energy associated with the atom laser. Following Eqs. (24) and (25), we obtain

$$
S(R)=\frac{\hbar}{2}\left[R \sqrt{R^{2}+\epsilon}+\epsilon \ln \left(\frac{R+\sqrt{R^{2}+\epsilon}}{\sqrt{-\epsilon}}\right)\right],
$$




$$
A(R)=\frac{\mathcal{B} \phi_{\mathrm{s}}\left(\mathbf{r}_{0}^{\prime}\right)}{\left[R^{2}\left(R^{2}+\epsilon\right)\right]^{1 / 4}} .
$$

$\mathcal{B}$ is proportional to the coupling strength and is not directly given by the eikonal treatment [60]. The atom laser beam amplitude $A(R)[61]$ is proportional to the BEC wave function value at the rf knife $\phi_{\mathrm{s}}\left(\mathbf{r}_{0}\right)$. The wave function at the BEC output surface is then

$$
\psi_{\ell}\left(R_{\mathrm{f}}\right)=A\left(R_{\mathrm{f}}\right) \exp \left[i S\left(R_{\mathrm{f}}\right) / \hbar\right] .
$$

\section{B. Propagation outside the condensate}

Once the matter wave has exited the condensate region, the volume source term $\rho$ vanishes and the beam wave function is given by the second term of Eq. (11) only, i.e., the Fresnel-Kirchhoff integral for matter waves, allowing one to compute the wave function at any point from the wave function on the BEC output surface. In this section, we calculate the propagation using an analytical expression for the timeindependent propagator and apply the $A B C D$ formalism in the paraxial regime.

\section{Fresnel-Kirchhoff Integral}

We perform the Fresnel-Kirchhoff integral in the $(x, z)$ plane at position $y_{0}$ :

$$
\psi_{\ell}=\frac{i \hbar}{2 m} \int_{\Gamma} d \mathbf{l}^{\prime}\left[\mathcal{G}_{E} \nabla \psi_{\ell}-\psi_{\ell} \nabla \mathcal{G}_{E}\right]
$$

where $\psi_{\ell}$ is nonzero only on the BEC output surface as seen in Sec. III A. The surface $\mathcal{S}$ of Eq. (11) is here reduced to its intersection contour $\Gamma$ with the vertical plane. It englobes the BEC volume and is closed at infinity.

Using the expression of $\mathcal{G}_{E}$ calculated in Appendix A, we compute Eq. (60) and the result is shown in Fig. 2 for four different outcoupling rf detunings. When coupling occurs at the top of the BEC, the propagation of the beam exhibits a strong divergence together with a well-contrasted interference pattern. The divergence is due to the strong expulsive potential experienced by the beam when crossing the condensate and interferences occur because atomic waves from different initial source points overlap during the propagation.

Comparison with a numerical Gross-Pitaevskii simulation shows good agreement. We also compare the results obtained by using at the BEC surface either Eq. (59) or Eq. (B9). The eikonal method fails when coupling at the very bottom of the BEC [Fig. 2(a)], since the classical turning point is too close to the BEC border, whereas the method using the exact solutions of the inverted harmonic potential agrees much better with the numerical simulation for any rf detuning. Finally, for very high coupling in the BEC [Fig. 2(d)], our model slightly overestimates the fringe contrast near the axis.

\section{Propagation in the paraxial regime}

Since the atom laser beam is accelerated by gravity, it enters quickly the paraxial regime. In the case considered in [39], the maximum transverse energy is given by the chemical potential $\mu$ whereas the longitudinal energy is mainly
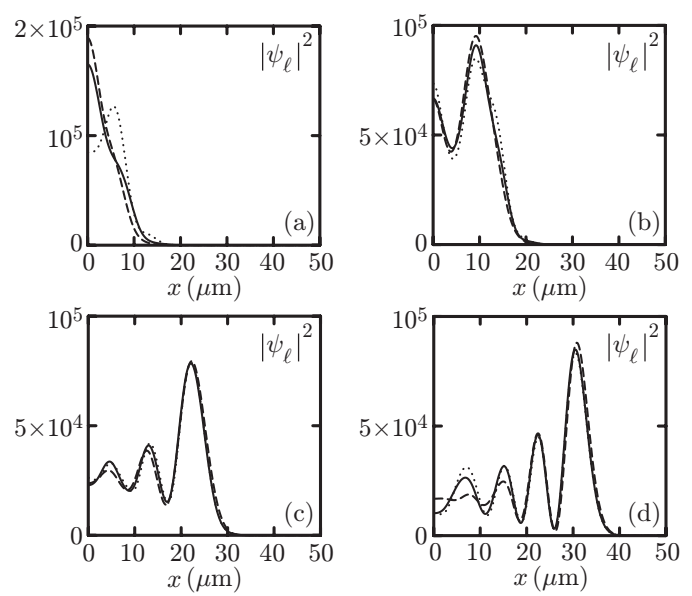

FIG. 2. Density profiles obtained at $150 \mu \mathrm{m}=z-\sigma$ below the BEC center. We consider the vertical plane $y_{0}=0$ and have normalized $\left|\psi_{\ell}\right|^{2}$ to unity. We have drawn the results obtained by using as input of the Kirchhoff integral the profile calculated using the eikonal [Eq. (59), dotted line] or exact solutions of the inverted harmonic oscillator [Eq. (B9), full line], and compare them to a full numerical integration of the two-dimensional Gross-Pitaevskii evolution of the atom laser (dashed line). The used rf detunings are (a) $\delta \nu=8900 \mathrm{~Hz}, \quad$ (b) $\delta \nu=6500 \mathrm{~Hz}$, (c) $\delta \nu=2100 \mathrm{~Hz}$, and (d) $\delta \nu=-1100 \mathrm{~Hz}$, and correspond to increasing outcoupling height, from (a) to (d).

related to the fall height $z$ by $E_{z} \approx m g z$. For $\mu$ typically of a few $\mathrm{kHz}$, one enters the paraxial regime after approximately $100 \mu \mathrm{m}$ of vertical propagation. For larger propagation distances, we can thus take advantage of the paraxial approximation presented in Sec. II B 4.

To proceed, we start from the profile $\psi_{\ell}(x)$ calculated after $150 \mu \mathrm{m}$ of propagation via the Kirchhoff integral. Using Eqs. (43)-(46), we extract the widths $\left\langle x x^{*}\right\rangle,\left\langle k k^{*}\right\rangle$ and the beam curvature $\mathcal{C}$ at this position. From these parameters we calculate the beam quality factor $M^{2}$ by using the general Eq. (49). Following the procedure presented in Sec. II B 4, we can choose the appropriate Hermite-Gauss decomposition of $\psi_{\ell}(x)$ and the propagation of each mode is then deduced from the $A B C D$ matrix corresponding to the transverse part of the potential described in Eq. (56): $V_{\perp}(x)=-(m / 2) \omega^{2} x^{2}$. The $A B C D$ matrix then reads

$$
\left(\begin{array}{ll}
A & B \\
C & D
\end{array}\right)=\left(\begin{array}{cc}
\cosh \omega\left(\zeta-\zeta^{\prime}\right) & \frac{\hbar}{m \omega} \sinh \omega\left(\zeta-\zeta^{\prime}\right) \\
\frac{m \omega}{\hbar} \sinh \omega\left(\zeta-\zeta^{\prime}\right) & \cosh \omega\left(\zeta-\zeta^{\prime}\right)
\end{array}\right)
$$

As explained in Sec. II B 4, the propagation is parametrized by the time $\zeta$, given by the classical equation of motion of the on-axis trajectory in the longitudinal part of the potential $V_{\|}(\widetilde{z})=-(m / 2) \omega^{2} \widetilde{z}^{2}$, where $\tilde{z}=z+\sigma_{q}$.

The $A B C D$ matrices formalism allows also to extract global propagation laws on the second order moments $X(\zeta), \quad K(\zeta)$ and evaluate the wavefront curvature $\mathcal{C}(\zeta)=\operatorname{Re}[K(\zeta) / X(\zeta)]$ associated with the wavefront $\psi_{\ell}(x, \zeta)$. 
By considering the paraxial evolution of the rms size $\sigma$ of $\psi_{\ell}(x, \zeta)$, we then obtain a generalized Rayleigh formula:

$$
\sigma^{2}(\xi)=\sigma_{0}^{2} \cosh ^{2}(\omega \xi)+\left(\frac{M^{2} \hbar}{2 m \omega}\right)^{2} \frac{\sinh ^{2}(\omega \xi)}{\sigma_{0}^{2}},
$$

involving the $M^{2}$ factor, and where $\sigma_{0}=X\left(\zeta_{0}\right)$ and $\xi=\zeta-\zeta_{0}$. We have introduced the focus time $\zeta_{0}$ so that $\mathcal{C}\left(\zeta_{0}\right)=0$. The relation (62) has been fruitfully used in [39] and [42] to extract the beam quality factor from experimental images.

\section{CONCLUSION}

Relying on the deep analogy between light waves and matter waves, we have introduced theoretical tools to deal with the propagation of coherent matter waves as follows.

The eikonal approximation is the standard treatment of geometrical optics. It is valid when diffraction, or wavepacket spreading, is negligible. It can be fruitfully used to treat short time propagation, as we show on the example of an atom laser beam crossing its source BEC.

The Fresnel-Kirchhoff integral comes from the classical theory of diffraction. It is particularly powerful as it allows to deal with piecewise defined potential in two or three dimensions together with taking into account diffraction and interference effects.

The $A B C D$ matrices formalism can be used as soon as the matter wave is in the paraxial regime. This widely used technique in laser optics provides simple algebraic laws to propagate the atomic wavefront, and also global laws on the second order moments of the beam, as the Rayleigh formula. Those results are especially suitable to characterize atom laser beams quality by the $M^{2}$ factor.

The toolbox developed in this paper can efficiently address a diversity of atom-optical setups in the limit where interactions in the laser remain negligible. It can be suited for beam focusing experiments $[62,63]$ and their potential application to atom lithography [64]. It also provides a relevant insight on beam profile effects in interference experiments involving atom lasers or to characterize the outcoupling of a matter-wave cavity [65]. It could also be used in estimating the coupling between an atom laser beam and a high finesse optical cavity [66]. Further developments may be carried out to generalize our work. In particular, the $M^{2}$ factor approach could be generalized to self interacting atom laser beam in the spirit of [67] or to more general cases of applications, such as non-paraxial beams or more complex external potential symmetries [68].

\section{ACKNOWLEDGMENTS}

The LCFIO and SYRTE are members of the Institut Francilien de Recherche sur les Atomes Froids (IFRAF). This work is supported by CNES (No. DA:10030054), DGA (Contracts No. 9934050 and No. 0434042), LNE, EU (Grants No. IST-2001-38863, No. MRTN-CT-2003-505032, and No. FINAQS STREP), and ESF (No. BEC2000+ and No. QUDEDIS).

\section{APPENDIX A: TIME-INDEPENDENT PROPAGATOR IN AN INVERTED HARMONIC POTENTIAL}

The time-dependent propagator of the inverted harmonic potential can be straightforwardly deduced from its expression for the harmonic potential [46] by changing real trapping frequencies to imaginary ones $(\omega \rightarrow i \omega)$. We derive here an analytic evaluation of its time-independent counterpart $\mathcal{G}_{E}$, by using the results of Sec. II B 2 .

We consider a potential in dimension $d$, characterized by the expulsing frequency $\omega$

$$
V(\mathbf{r})=V(\mathbf{0})-\sum_{j \in \llbracket 1 \ldots d \rrbracket} \frac{1}{2} m \omega^{2} r_{j}^{2} .
$$

By introducing the reduced time $s=\omega \tau$ and the harmonic oscillator size $\sigma_{\mathrm{o}}=\sqrt{\hbar / m \omega}, \mathcal{G}_{E}$ is expressed as

$$
\mathcal{G}_{E}\left(\mathbf{r}, \mathbf{r}^{\prime}\right)=\int_{0}^{\infty} d s \mathcal{H}(s) e^{i \phi\left(\mathbf{r}, \mathbf{r}^{\prime}, s\right)},
$$

with $\mathcal{H}(s)=m /(2 \pi i \hbar \sinh s)$, and

$$
\phi=\frac{\left[\left(\mathbf{r}^{2}+\mathbf{r}^{\prime 2}\right) \cosh s-2 \mathbf{r} \cdot \mathbf{r}^{\prime}\right]}{2 \sigma_{\mathrm{o}}^{2} \sinh s}+\frac{[E-V(\mathbf{0})]}{\hbar \omega} s .
$$

The first-order stationary times $s_{ \pm}$verify

$$
\cosh s_{ \pm}=\frac{-b \pm \sqrt{b^{2}+4[E-V(\mathbf{0})] c}}{2[E-V(\mathbf{0})]},
$$

where $b=m \omega^{2} \mathbf{r} \cdot \mathbf{r}^{\prime}$ and $c=E-V(0)+m \omega^{2}\left(\mathbf{r}^{2}+\mathbf{r}^{\prime 2}\right) / 2$. If there are positive and real solutions $s_{ \pm}, \mathcal{G}_{E}$ reads [Eq. (16)]

$$
\mathcal{G}_{E}^{(1)}\left(\mathbf{r}, \mathbf{r}^{\prime}\right)=\sum_{s_{ \pm}>0} \sqrt{\frac{2 i \pi}{\partial^{2} \phi /\left.\partial s^{2}\right|_{s_{ \pm}}}} \mathcal{H}\left(s_{ \pm}\right) e^{i \phi\left(s_{ \pm}\right)} \text {. }
$$

Otherwise, the relevant stationary point $s_{0}$ [Eq. (19)] verifies

$$
\cosh s_{0}=\frac{\mathbf{r}^{2}+\mathbf{r}^{\prime 2}+\sqrt{\left(\mathbf{r}+\mathbf{r}^{\prime}\right)^{2}\left(\mathbf{r}-\mathbf{r}^{\prime}\right)^{2}}}{2 \mathbf{r} \cdot \mathbf{r}^{\prime}}
$$

$s_{0}$ is the time associated with the classical trajectory connecting $\mathbf{r}^{\prime}$ and $\mathbf{r}$ with the closest energy to $E$. If the angle between $\mathbf{r}$ and $\mathbf{r}^{\prime}$ is above $\pi / 2$ then, according to Eq. (A6), the absolute value of the first derivative of $\phi$ is never minimal, so that $e^{i \phi(s)}$ quickly oscillates over $[0 ;+\infty)$ and one can take $\mathcal{G}_{E}\left(\mathbf{r}, \mathbf{r}^{\prime}\right)=0$. In other cases, where the solution is unique, one develops the phase around $s_{0}$ and $\mathcal{G}_{E}$ finally expresses as [Eq. (20)]

$$
\mathcal{G}_{E}^{(2)}\left(\mathbf{r}, \mathbf{r}^{\prime}\right)=\frac{2 \pi \mathcal{H}\left(s_{0}\right)}{\kappa} e^{i \phi\left(s_{0}\right)} \mathrm{Ai}\left(-\left.\frac{1}{\kappa} \frac{\partial \phi}{\partial s}\right|_{s_{0}}\right),
$$

where $\kappa=\left[-\left.(1 / 2)\left(\partial^{3} \phi / \partial s^{3}\right)\right|_{s_{0}}\right]^{1 / 3}$.

\section{APPENDIX B: EXACT SOLUTIONS OF THE TWO- DIMENSIONAL INVERTED HARMONIC OSCILLATOR AND RELATION WITH THE EIKONAL}

In this appendix, we give an analytical expression for the eigenfunctions of the inverted harmonic potential in the BEC 
region. The use of such solutions enables us to avoid any divergence of the eikonal solution close to the turning point.

Using dimensionless parameters introduced in Eq. (57), the time-independent Schrödinger equation in the BEC region reads

$$
-\left(\frac{\partial^{2} \psi}{\partial R^{2}}+\frac{1}{R} \frac{\partial \psi}{\partial R}+\frac{1}{R^{2}} \frac{\partial^{2} \psi}{\partial \alpha^{2}}\right)-R^{2} \psi=\epsilon \psi .
$$

Introducing the angular momentum $L_{\alpha}=(\hbar / i)(\partial \psi / \partial \alpha)$, one can decompose the solution of this equation as the product of a radial part and an angular part

$$
\psi(R, \alpha)=\phi(R) e^{i l \alpha},
$$

with $l \in \mathbb{Z}$ and $\hbar l$ is the angular momentum of the wave function. The general solution $\phi$ is given by

$$
\phi(R)=\frac{c_{1}}{R} \mathrm{M}\left(-i \frac{\epsilon}{4} ; \frac{l}{2} ; i R^{2}\right)+\frac{c_{2}}{R} \mathrm{~W}\left(-i \frac{\epsilon}{4} ; \frac{l}{2} ; i R^{2}\right) .
$$

$\mathrm{M}(\mu, \nu, z)$ and $\mathrm{W}(\mu, \nu, z)$ are Whittaker functions (related to the confluent hypergeometric functions of the first and second kind) [49], whereas $c_{1}$ and $c_{2}$ are complex coefficients.

In general, the wave function must be decomposed on the basis of the different solutions $\phi(R)$ parametrized by $l$ and $\epsilon$. However, in the following, we restrict ourselves to the study of a solution that connects asymptotically to the eikonal. Thus we are only interested in the wave function describing a dynamics without any transverse speed or diffraction, i.e., with $l=0$. Since the wave progresses from the rf knife $R_{0}$ to the outer part of the potential, we also only look for "outgoing wave" type solutions [69]. Such solutions behave as progressive waves in the asymptotic limit $(R \rightarrow \infty)$. One can express the Whittaker functions in term of hypergeometric functions [49] for any complex parameter $\mu$ and $z$

$$
\mathrm{M}(\mu, 0, z)=e^{-z / 2} \sqrt{z}{ }_{1} \mathrm{~F}^{1}\left(\frac{1}{2}-\mu ; 1 ; z\right),
$$

$$
\mathrm{W}(\mu, 0, z)=e^{-z / 2} z^{\mu}{ }_{2} \mathrm{~F}^{0}\left(\frac{1}{2}-\mu, \frac{1}{2}-\mu ; ;-\frac{1}{z}\right) .
$$

For $|z| \rightarrow \infty$, these functions are asymptotically expanded as [70]

$$
\begin{aligned}
{ }_{1} \mathrm{~F}^{1}(a ; b ; z) \sim & \frac{\Gamma(b)}{\Gamma(b-a)}(-z){ }_{2}{ }_{2} \mathrm{~F}^{0}\left(a, a-b+1 ; ;-\frac{1}{z}\right) \\
& +\frac{\Gamma(b)}{\Gamma(a)} e^{z} z^{a-b}{ }_{2} \mathrm{~F}^{0}\left(b-a, 1-a ; ; \frac{1}{z}\right)
\end{aligned}
$$

and

$$
{ }_{2} \mathrm{~F}^{0}\left(a, b ; ; \frac{1}{z}\right) \rightarrow 1+O\left(\frac{1}{z}\right) .
$$

One thus obtains an asymptotic formula for Eq. (B3) in which terms proportional to $e^{i R^{2} / 2}$ or $e^{-i R^{2} / 2}$ appear. Cancelling the second ones corresponding to an incoming wave toward the center leads to a relation between $c_{1}$ and $c_{2}$ :

$$
i \frac{e^{-\pi \epsilon / 4}}{\Gamma\left(\frac{1}{2}-i \frac{\epsilon}{4}\right)} c_{1}+c_{2}=0
$$

The solution is finally written as

$$
\begin{aligned}
\psi(R)= & \frac{\Gamma\left(\frac{1}{2}+i \frac{\epsilon}{4}\right) e^{i \epsilon[1-\ln (-\epsilon / 4)] / 4}}{R}\left[e^{\pi \epsilon / 8} \mathrm{M}\left(-i \frac{\epsilon}{4} ; 0 ; i R^{2}\right)\right. \\
& \left.-\frac{i e^{-\pi \epsilon / 8}}{\Gamma\left(\frac{1}{2}-i \frac{\epsilon}{4}\right)} \mathrm{W}\left(-i \frac{\epsilon}{4} ; 0 ; i R^{2}\right)\right]
\end{aligned}
$$

where the prefactor has been chosen so that the asymptotic expression of $\psi(R)$ connects to the eikonal solution given by Eq. (58).
[1] See, for example, Quantum Mechanics for Space Application: From Quantum Optics to Atom Optics and General Relativity, special issue of Appl. Phys. B 84 (4) (2006).

[2] M.-O. Mewes, M. R. Andrews, D. M. Kurn, D. S. Durfee, C. G. Townsend, and W. Ketterle, Phys. Rev. Lett. 78, 582 (1997).

[3] B. P. Anderson and M. A. Kasevich, Science 282, 1686 (1998).

[4] I. Bloch, T. W. Hänsch, and T. Esslinger, Phys. Rev. Lett. 82, 3008 (1999).

[5] E. W. Hagley, L. Deng, M. Kozuma, J. Wen, K. Helmerson, S. L. Rolston, and W. D. Phillips, Science 283, 1706 (1999).

[6] G. Cennini, G. Ritt, C. Geckeler, and M. Weitz, Phys. Rev. Lett. 91, 240408 (2003).

[7] N. P. Robins, C. Figl, S. A. Haine, A. K. Morrison, M. Jeppesen, J. J. Hope, and J. D. Close, Phys. Rev. Lett. 96, 140403 (2006).

[8] W. Guerin, J.-F. Riou, J. P. Gaebler, V. Josse, P. Bouyer, and A.
Aspect, Phys. Rev. Lett. 97, 200402 (2006).

[9] C. J. Bordé, C. R. Acad. Sci., Ser. IV Phys. Astrophys. 2, 509 (2001).

[10] P. Storey and C. Cohen-Tannoudji, J. Phys. II 4, 1999 (1994).

[11] C. Antoine and C. J. Bordé, J. Opt. B: Quantum Semiclassical Opt. 5, S199 (2003); C. J. Bordé, Gen. Relativ. Gravit. 36, 475 (2004).

[12] K. Bongs, R. Launay, and M. A. Kasevich, Appl. Phys. B: Lasers Opt. 84, 599 (2006).

[13] G. Wilpers, C. W. Oates, and L. Hollberg, Appl. Phys. B: Lasers Opt. 85, 31 (2006).

[14] G. Wilpers et al., Metrologia 44, 146 (2007).

[15] R. J. Ballagh, K. Burnett, and T. F. Scott, Phys. Rev. Lett. 78, 1607 (1997).

[16] M. Naraschewski, A. Schenzle, and H. Wallis, Phys. Rev. A 56, 603 (1997).

[17] H. Steck, M. Naraschewski, and H. Wallis, Phys. Rev. Lett. 80, 1 (1998). 
[18] W. Zhang and D. F. Walls, Phys. Rev. A 57, 1248 (1998).

[19] J. Schneider and A. Schenzle, Appl. Phys. B: Lasers Opt. 69, 353 (1999).

[20] Y. B. Band, P. S. Julienne, and M. Trippenbach, Phys. Rev. A 59, 3823 (1999).

[21] M. Edwards, D. A. Griggs, P. L. Holman, C. W. Clark, S. L. Rolston, and W. D. Phillips, J. Phys. B 32, 2935 (1999).

[22] R. Graham and D. F. Walls, Phys. Rev. A 60, 1429 (1999).

[23] J. Schneider and A. Schenzle, Phys. Rev. A 61, 053611 (2000).

[24] N. P. Robins, C. M. Savage, J. J. Hope, J. E. Lye, C. S. Fletcher, S. A. Haine, and J. D. Close, Phys. Rev. A 69, 051602(R) (2004).

[25] N. P. Robins, A. K. Morrison, J. J. Hope, and J. D. Close, Phys. Rev. A 72, 031606(R) (2005).

[26] B. Kneer, T. Wong, K. Vogel, W. P. Schleich, and D. F. Walls, Phys. Rev. A 58, 4841 (1998).

[27] N. Robins, C. Savage, and E. A. Ostrovskaya, Phys. Rev. A 64, 043605 (2001).

[28] F. Gerbier, P. Bouyer, and A. Aspect, Phys. Rev. Lett. 86, 4729 (2001); 93, 059905(E) (2004).

[29] G. M. Moy and C. M. Savage, Phys. Rev. A 56, R1087 (1997).

[30] G. M. Moy, J. J. Hope, and C. M. Savage, Phys. Rev. A 59, 667 (1999)

[31] M. W. Jack, M. Naraschewski, M. J. Collett, and D. F. Walls, Phys. Rev. A 59, 2962 (1999).

[32] A. S. Bradley, J. J. Hope, and M. J. Collett, Phys. Rev. A 68, 063611 (2003).

[33] Y. Japha, S. Choi, K. Burnett, and Y. B. Band, Phys. Rev. Lett. 82, 1079 (1999).

[34] S. Choi, Y. Japha, and K. Burnett, Phys. Rev. A 61, 063606 (2000).

[35] Y. Japha and B. Segev, Phys. Rev. A 65, 063411 (2002).

[36] J. Ruostekoski, T. Gasenzer, and D. A. W. Hutchinson, Phys. Rev. A 68, 011604(R) (2003).

[37] T. Busch, M. Köhl, T. Esslinger, and K. Mølmer, Phys. Rev. A 65, 043615 (2002); 65, 069902(E) (2002).

[38] T. Kramer and M. Rodríguez, Phys. Rev. A 74, 013611 (2006); 75, 069905(E) (2007).

[39] J.-F. Riou, W. Guerin, Y. Le Coq, M. Fauquembergue, V. Josse, P. Bouyer, and A. Aspect, Phys. Rev. Lett. 96, 070404 (2006).

[40] Y. Le Coq, J. H. Thywissen, S. A. Rangwala, F. Gerbier, S. Richard, G. Delannoy, P. Bouyer, and A. Aspect, Phys. Rev. Lett. 87, 170403 (2001).

[41] R. G. Dall, L. J. Byron, A. G. Truscott, G. R. Dennis, M. T. Johnsson, M. Jeppesen, and J. J. Hope, Opt. Express 15, 17673 (2007).

[42] M. Jeppesen, J. Dugué, G. R. Dennis, M. T. Johnsson, C. Figl, N. P. Robins, and J. D. Close, e-print arXiv:0704.0291v2.

[43] G. Barton, Elements of Green's Functions and Propagation (Clarendon Press, Oxford, 1989).

[44] C. J. Bordé, in Fundamental Systems in Quantum Optics, edited by J. Dalibard, J. M. Raimond, and J. Zinn-Justin, Proceedings of the Les Houches Summer School of Theoretical Physics, Session LIII (Elsevier Science Publishers B. V., Am- sterdam, 1990).

[45] M. Born and E. Wolf, Principles of Optics, 7th ed. (Cambridge University Press, Cambridge, UK, 2002).

[46] R. P. Feynman and A. R. Hibbs, Quantum Mechanics and Path Integrals (McGraw-Hill, New York, 1965).

[47] J. H. van Vleck, Proc. Natl. Acad. Sci. U.S.A. 14, 178 (1928).

[48] L. Brillouin, Ann. Sci. Ec. Normale Super. 33, 17 (1916).

[49] M. Abramowitz and I. E. Stegun, Handbook of Mathematical Functions (Dover, New York, 1972).

[50] A. Messiah, Quantum Mechanics (Dover, New York, 2000).

[51] L. D. Landau and E. M. Lifchitz, The Classical Theory of Fields, 4th ed. (Pergamon Press, Oxford, England, 1975).

[52] The stationary phase method could also provide a way to calculate this integral; see [59].

[53] A. E. Siegman, in Solid State Lasers: New Developments and Applications, edited by M. Inguscio and R. Wallenstein (Plenum Press, New York, 1993).

[54] P. A. Bélanger, Opt. Lett. 16, 196 (1991).

[55] A. E. Siegman, IEEE J. Quantum Electron. 27, 1146 (1991).

[56] F. Dalfovo, S. Giorgini, L. Pitaevskii, and S. Stringari, Rev. Mod. Phys. 71, 463 (1999).

[57] M. Köhl, T. Busch, K. Mølmer, T. W. Hänsch, and T. Esslinger, Phys. Rev. A 72, 063618 (2005).

[58] This has been checked in a similar configuration by numerical simulations as reported in [57].

[59] J.-F. Riou, Ph.D. thesis, Institut d'Optique, 2006.

[60] A detailed development of the first term of Eq. (11) using the stationary phase approximation leads to the value $\mathcal{B}$ $=\Omega_{\mathrm{R}} / \omega_{\perp} \sqrt{i \pi / 2}$ [59]. However, in practice, as long as one is interested only in the shape of the wave function, the precise value of $C$ is not necessary.

[61] This treatment does not allow us to predict the wave function value in the vicinity of the classical radial turning point $R_{0}$ $=\sqrt{-\epsilon}$ since the normalization $A(R)$ diverges as $R \rightarrow R_{0}$. A more accurate treatment is thus needed to deal with the few trajectories starting at the edge of the rf knife. One possibility is to use the exact solution $\psi$ of the radially symmetric twodimensional inverted potential, as presented in Eq. (A7).

[62] I. Shvarchuck, Ch. Buggle, D. S. Petrov, K. Dieckmann, M. Zielonkowski, M. Kemmann, T. Tiecke, W. von Klitzing, G. V. Shlyapnikov, and J. T. M. Walraven, Phys. Rev. Lett. 89, 270404 (2002).

[63] A. S. Arnold, C. MacCormick, and M. G. Boshier, J. Phys. B 37, 485 (2004).

[64] E. Knyazchyan, B. Mercier, H. Perrin, P.-E. Pottie, and V. Lorent, J. Phys.: Conf. Ser. 19, 44 (2005).

[65] F. Impens, P. Bouyer, and C. J. Bordé, Appl. Phys. B: Lasers Opt. 84, 603 (2006).

[66] S. Ritter, A. Öttl, T. Donner, T. Bourdel, M. Köhl, and T. Esslinger, Phys. Rev. Lett. 98, 090402 (2007).

[67] C. Paré and P. A. Bélanger, Opt. Quantum Electron. 24, S1051 (1992).

[68] F. Impens, Phys. Rev. A 77, 013619 (2008).

[69] H. A. Fertig and B. I. Halperin, Phys. Rev. B 36, 7969 (1987).

[70] L. D. Landau and E. M. Lifchitz, Quantum Mechanics, 3rd ed. (Pergamon Press, Oxford, England, 1977). 